\title{
Antioxidant effects of potassium ascorbate with ribose therapy in a case with Prader Willi Syndrome
}

\author{
C. Anichini, F. Lotti, M. Longini, F. Proietti, C. Felici, S. Perrone and G. Buonocore* \\ Department of Pediatrics, Obstetrics and Reproductive Medicine, University of Siena, Siena, Italy
}

\begin{abstract}
Oxidative stress (OS) is involved in several human diseases, including obesity, diabetes, atherosclerosis, carcinogenesis, as well as genetic diseases. We previously found that OS occurs in Down Syndrome as well as in Beckwith-Wiedemann Syndrome (BWS). Here we describe the clinical case of a female patient with Prader Willi Syndrome (PWS), a genomic imprinting disorder, characterized by obesity, atherosclerosis and diabetes mellitus type 2, pathologies in which a continuous and important production of free radicals takes place. We verified the presence of OS by measuring a redox biomarkers profile including total hydroperoxides $(\mathrm{TH})$, non protein-bound iron (NPBI), thiols (SH), advanced oxidation protein products (AOPP) and isoprostanes (IPs). Thus we introduced in therapy an antioxidant agent, namely potassium ascorbate with ribose (PAR), in addition to GH therapy and we monitored the redox biomarkers profile for four years. A progressive decrease in OS biomarkers occurred until their normalization. In the meantime a weight loss was observed together with a steady growth in standards for age and sex.
\end{abstract}

Keywords: Prader Willi Syndrome, oxidative stress, potassium ascorbate with ribose, isoprostanes

\section{Introduction}

Oxidative stress (OS) injury occurs when tissues, cells and biomolecules undergo an excessive exposition to oxidizing agents, above all free radicals (FRs) [7, $10]$. It is associated with a large number of human pathologies, such as carcinogenesis, cardiovascular and neurodegenerative disease $[11,20]$. OS also has a relevant role in pediatric and neonatal pathologies including retinopathy of prematurity, necrotizing enterocolitis, intraventricular hemorrhage, periventricular leukomalacia [28,29,33].

Currently it is widely accepted that OS is involved in the pathogenesis of Down Syndrome. OS may be responsible for the neural damage observed in the patients, as well as the other common clinical pathologies, such as leukemia, endocrine dysfunctions, early ageing $[4,9,25,26]$.

${ }^{*}$ Corresponding author: Giuseppe Buonocore, Department of Pediatrics, Obstetrics and Reproductive Medicine, University of Siena, Viale Mario Bracci 36, 53100 Siena, Italy. Tel.: +39 0577586 523; Fax: +39 0577586 143; E-mail: giuseppe.buonocore@unisi.it.
Here we tested the hypothesis that OS occurs in Prader Willi Syndrome (PWS), a genomic imprinting disorder characterized by obesity, atherosclerosis and diabetes mellitus type 2 , pathologies in which a continuous and important production of FRs takes place. PWS is due to an anomaly of chromosome 15 with loss of the paternal copy of the region 15q11-13 [3]: this condition can occur as a consequence of a deletion (70\% of patients), of a maternal uniparental disomy (25\% of patients), or of an imprinting centre defect (5\% of patients) $[16,18,22,23]$. One of the most important features of PWS is obesity, caused by an insatiable hunger which emerges in affected children aged 2-6 years [12,17,30]: it is responsible for complications such as atherosclerosis and diabetes mellitus type 2, pathologies in which OS occurs. In fact, atherosclerosis is at present considered a chronic inflammatory disease, in which a continuous and important production of FRs takes place [6,27]. In diabetes, hyperglycemia causes an increased mithochondrial production of superoxide radical which leads to a greater cellular exposition to ROSs [14,19]. 
Other clinical features of PWS are generalized hypotonia in newborns, with poor suck, feeding problems and failure to thrive after birth and, later on, bulimia, that, together with a residual hypotonia, causes the severe obesity. PWS patients have a typical facies, characterized by almond-shaped eyes, narrow bifrontal diameter, narrow nasal bridge, thin upper lip with downturned mouth; there are also short stature, due to a growth hormone deficiency, central hypogonadism, delayed psychomotor development, language issues, mild mental retardation, behavioral and psychiatric disturbances, sleep abnormalities [2,5,15,31].

Here we describe the clinical case of a female patient with PWS, in which, after the verification of a ongoing OS, we tested the antioxidant effects of potassium ascorbate with ribose (PAR), administered together with GH therapy. Potassium ascorbate is a salt derived from natural ascorbic acid, totally non-toxic, that has antioxidant effects combining the antioxidant action of vitamin $\mathrm{C}$ with the stabilizing intracellular effects of potassium. The ribose acts as a catalyst strengthening the action of potassium ascorbate [32].

\section{Clinical report}

The patient is a girl aged 13 years. She was born in August 1999, at 33 weeks of gestation by cesarean section. Neurological features at birth were characterized by generalized hypotonia, poor reflexes, weak cry and poor suck. Since the first hospitalization, some typical facial features induced to the clinical suspect of PWS, and genetic analyses were performed. The presence of the deletion in the paternal copy of chromosome 15 was demonstrated by using the FISH test. The patient was periodically evaluated according to a PWS management program.

Since the first controls a growth delay emerged: height and weight were far below the growth standards. After the demonstration of a reduced basal secretion of growth hormone, in January 2002 the replacement therapy was started with an initial dose of $1.5 \mathrm{mg} / \mathrm{m}^{2} /$ day for 6 days per week; afterwards, in January 2007, the dose was doubled and taken from $0.6 \mathrm{mg} /$ day to $1.2 \mathrm{mg} /$ day. The trend of growth curves both for height and for weight showed a good response to therapy: at present the girl's height is between 10th and 25 th centile, falling into growth standards for age and sex.

Starting from March 2006, a redox biomarkers profile was tested and monitored to verify the effects of the antioxidant therapy with PAR.

\section{Materials and methods}

OS was detected by measuring a redox panel represented by total hydroperoxides $(\mathrm{TH})$, non proteinbound iron (NPBI), thiols ( $\mathrm{SH}$ ), advanced oxidation protein products (AOPP) and isoprostanes.

Isoprostanes are generated during the peroxidation of the arachidonic acid; they are considered the most specific markers of oxidative stress (n.v. $<60 \mathrm{pg} / \mathrm{ml}$ ). They were measured according to the method of Milne et al. [21]. Quantification of IsoPs requires two purification steps using solid-phase C-18 Sep-Pak cartridge followed by silica Sep-Pak cartridge. After the extraction these molecules are converted to the corresponding pentafluorobenzyl esters to facilitate compound analysis by GC-NICI-MS. Pentafluorobenzyl esters were purified by TLC. The purified compounds were converted to the corresponding trimethylsilyl ether. The sample dissolved in $10 \mu \mathrm{L}$ of undecane is injected into GC/MS. The values of OS markers were expressed in $\mathrm{pg} / \mathrm{ml}$ of plasma. Simultaneous determination of AOPP could provide information regarding an aspect of proteins involvement in FRs reactions, namely oxidized plasma proteins that have lost their oxidant properties. AOPP were measured as described by Witko-Sarsat et al. [34]. The method is based on the reaction of AOPP with Potassium Iodure in acidic condition, the result is measured by a spectrophotometric reading at $340 \mathrm{~nm}$. AOPP concentrations were expressed as micromolar chloramines-T equivalents (n.v. $<29 \pm 0.49 \mu \mathrm{mol} / \mathrm{L}$ ).

$\mathrm{TH}$ represent a measure of overall OS, given that they are the intermediate oxidative products of lipids, peptides and aminoacids. TH production was measured with a d-ROMs Kit by Diacron Srl, Grosseto, Italy (n.v. included between 250 and 300 CARR U - Carratelli Units- 1 CARR $U$ is equivalent to $0.08 \mathrm{mg} / \mathrm{L}$ of $\mathrm{H}_{2} \mathrm{O}_{2}$ ).

NPBI is a pro-oxidant substance able to act as a catalyst of Fenton reaction that produces free radicals. Usually in physiological conditions it is not detectable. We measured it according to our previous published HPLC method [24].

Thiols (SH groups) represent protective factors: they keep the antioxidant system and the proteins in the reduced state; they are measured according to the method of $\mathrm{Hu}$ et al. [13]. Briefly, the method is based on the reaction of $\mathrm{SH}$ groups with Dithio-bis (2nitrobenzoic acid). The resulting color is measured by spectrophotometer at a wavelength of $412 \mathrm{~nm}$ (n.v. 450-650 $\mu \mathrm{mol} / \mathrm{L})$.

All the parameters were monitored about every 6 months. The first evaluation of the redox biomarkers 


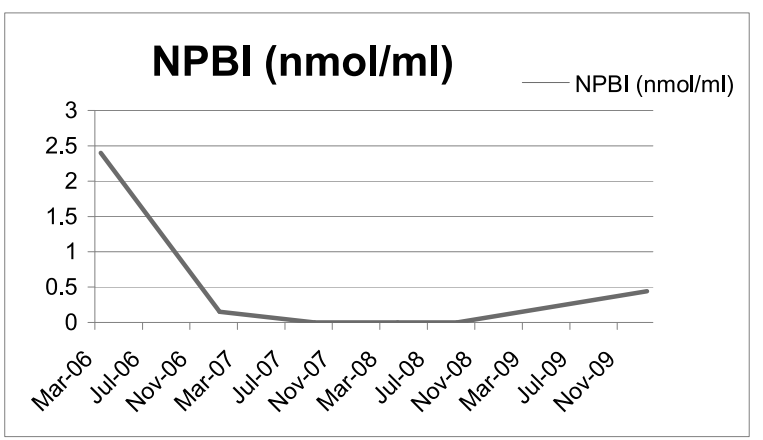

Fig. 1. Trend of NPBI in the patient (n.v.: absent).

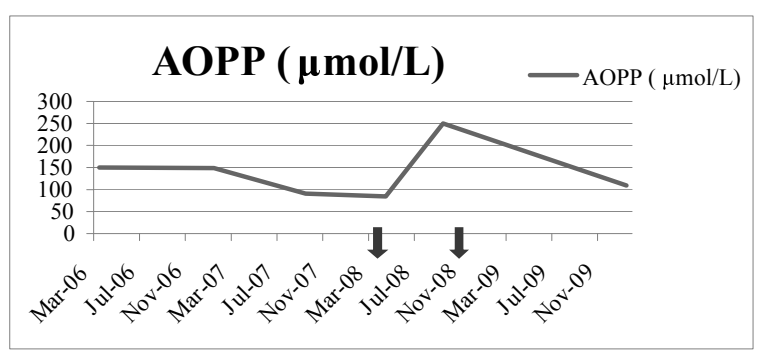

Fig. 2. Trend of AOPP in the patient (n.v.: $20 \mu \mathrm{mol} / \mathrm{L}$ ). The arrows show the beginning and the end of the period of suspension of the therapy. Note the worsening during the temporary stop and the quick improvement when the therapy was restored.

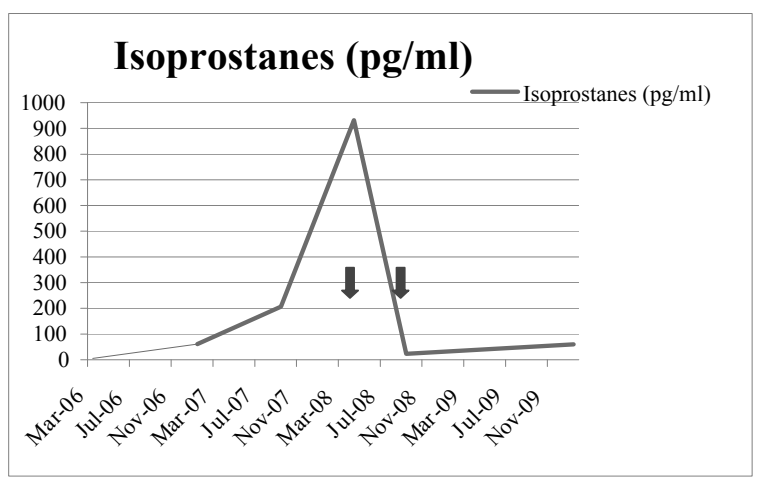

Fig. 3. Trend of Isoprostanes in the patient (n.v. $<60 \mathrm{pg} / \mathrm{ml}$ ). The arrows show the beginning and the end of the period of suspension of the therapy. Note the worsening during the temporary stop and the quick improvement when the therapy was restored.

profile in our patient was made in March 2006, when the patient was 6,7 years old. The results showed a clear abnormal redox pattern indicating the presence of OS. Thus we introduced in therapy PAR, an antioxidant agent which previously showed positive effects in BWS [1].

Heparinized blood samples were drawn and immediately centrifuged (1000xg. for minutes 10$)$. The plasma

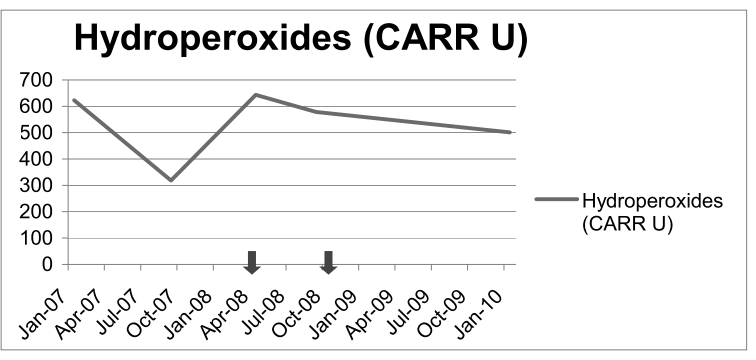

Fig. 4. Trend of Hydroperoxides in the patient (n.v. 250-300 CARR $\mathrm{U})$. The arrows show the beginning and the end of the period of suspension of the therapy. Note the worsening during the temporary stop and the quick improvement when the therapy was restored.

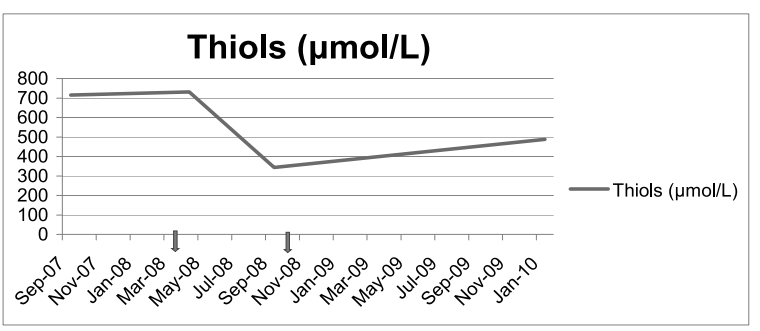

Fig. 5. Trend of Thiols in the patient (n.v. $>450 \mu \mathrm{mol} / \mathrm{L}$ ). The arrows show the beginning and the end of the period of suspension of the therapy. Note the worsening during the temporary stop and the improvement when the therapy was restored. Thiols represent protective factors: they are a marker for internal body's defences and it is acceptable that they remain relatively low after reintroduction of therapy.

was stored in plastic metal-free containers at $-80^{\circ} \mathrm{C}$ until analyses, which were performed at the Laboratory of Oxidative Stress of the University of Siena. During the whole period of observation no changes in the environment or in the lifestyle of the patient occurred.

As antioxidant agent we used PAR, administering a daily dose of: L-Ascorbic Acid $150 \mathrm{mg}$, D-Ribose $3 \mathrm{mg}$, Potassium Bicarbonate $300 \mathrm{mg}$, on the basis of a protocol used in other genetic syndromes and approved by the local Ethics Committee of University Hospital of Siena. The dosage used is the minimal dosage for the prevention of degenerative disorders in adults.

\section{Results and follow up}

Ten months after the administration of PAR (January 2007), the redox biomarkers panel showed reduced values. NPBI concentrations showed a trend in decreasing in parallel with an increase in the values of thiols. The efficacy of the treatment was confirmed by the follow up. Little is known about the role of OS in PWS and no data exist about OS biomarkers in the PWS. In our case 
it is interesting to note that a considerable worsening of the redox profile was observed when the patient's mother voluntarily decided to suspend the therapy during the summertime believing that a vitamin supplement was not necessary due to the variety of fruits in the summer diet. The redox profile was back to pre-suspension values when the therapy was restored (see Figs 1-5). Therefore the temporary unexpected stop of therapy likely adds an extra proof for the efficacy of PAR. No side effects, nor ingestion problems were detected.

\section{Discussion and conclusions}

In our patient we supposed the presence of OS. The syndrome is, indeed, the most important genetic cause of obesity, that is a risk factor for atherosclerosis and that is usually linked to hyperlipidemia, arterial hypertension and diabetes mellitus type 2, conditions in which OS occurs. Obesity in PWS is the consequence of the uncontrollable hyperphagia, for which an effective pharmacological treatment is not available at present; consequently the only useful therapy for obesity is bariatric surgery. It is noteworthy that OS is emerging as an important degenerative mechanism at a cerebral level (a correlation has been demonstrated between free radicals and neurodegenerative diseases such as Parkinson's disease and ataxic syndromes) [8]. Thus we supposed that an antioxidant treatment with PAR might modulate at the CNS level the mechanism responsible for bulimia.

In association to the reduction of OS obtained in our patient, we observed a positive effect on her phenotype. In fact, the most interesting aspect of this clinical case is probably the lack of the characteristic obesity: the patient's weight remained stable below the 25 th centile. Obviously other factors may have influenced this result, above all the early diagnosis and the early GH therapy. The positive effects of GH therapy were related to a better growth rate with an improvement in body composition that is reduction of fat mass and increase of lean mass. Little is known, few and contrasting data have been reported about the antioxidant or oxidative effects of GH therapy in animals. We have no data about the effects on OS of GH therapy in our patient, because the evaluation of OS was performed only at a later time, after the introduction of $\mathrm{GH}$.

The clinical controls of the patient included thyroid function monitoring, for the frequent finding of a secondary hypothyroidism in the syndrome, and the lipidic and glycemic profiles. We checked the cardiorespirato- ry function with ECG, echocardiogram and spirometry and sleep problems with polysomnography. The patient also underwent psychological and logopedic evaluations for behavioral and language issues. She showed significant improvements in all these aspects.

In conclusion, three points emerge in our PWS patient: firstly, the presence of OS. Secondly, the beneficial effect of PAR therapy. Thirdly, PAR seems to be particularly promising since not only is inexpensive and has no collateral effects, but it seems indeed to give positive and encouraging results on clinical features. It is noteworthy that PAR gave important and interesting results also in other genetic syndromes, such as the Beckwith-Wiedemann Syndrome [1].

Obviously this is only a case report and further research will be needed to confirm the utility of PAR therapy in Prader Willi Syndrome. Our results pave the way for larger studies recruiting more patients at pediatric as well as at adult age. Early diagnosis, with careful evaluation of suspect cases, is still of great importance, in order to start a carefully structured treatment as soon as possible, avoiding unnecessary interventions and engaging, instead, suitable clinical and rehabilitative steps.

\section{Acknowledgments}

This work was supported partially by Europe Against Infant Brain Injury and by funds for research of the University of Siena awarded to Dr. Anichini for 20062007. We thank the family involved in the study for its collaboration. The authors have no relevant financial relationships to disclose or conflict of interest to resolve.

\section{References}

[1] Anichini C, Lo Rizzo C, Longini M, Paoli G, Di Bartolo RM, Proietti F, Buonocore G. beckwith-Wiedemann Syndrome: Potassium Ascorbate with Ribose Therapy in a Syndrome with High Neoplastic Risk. Anticancer Research. 2011 Nov; 31(11): 3973-6.

[2] Aughton DJ, Cassidy SB. Physical features of Prader Willi syndrome. Am J Dis Child. 1990; 144: 1251-1254.

[3] Beccaria L, Bosio L, Benzi F, Bregoni P, Achutegui I, Chiumello G, Livieri C, Trifirò G, De Toni T, Iughetti L, Ragusa L, Salvatoni A, Tonini G, Corrias A, Crinò A. Prader Willi syndrome. Ann Ist Super Sanità. 1999; 35(2): 221-232.

[4] Busciglio J, Yankner BA. Apoptosis and increased generation of reactive oxygen species in Down's syndrome neurons in vitro. Nature. 1995; 378: 776-779. 
[5] Cassidy SB, Morris CA. Behavioral phenotypes in genetic syndromes: genetic clues to human behavior. Adv Pediatr. 2002; 49: 59-86.

[6] Chobanyan Jürgens K, Pham VV, Stichtenoth DO, Tsikas D. Asymmetrical Dimethylarginine, Oxidative Stress and Atherosclerosis. Hypertension. 2011 Nov; 58(5): e184-5.

[7] Del Maestro R. An approach to free radicals in medicine and biology. Acta Physiol Scand. 1992; 492: 153-168.

[8] Farooqi T, Farooqi AA. Lipid-mediated oxidative stress and inflammation in the pathogenesis of Parkinson's disease. Parkinson's Dis. 2011 Feb 15; 2011: 247467.

[9] Groner Y, Elroy-Stein O, Avraham KB, Schickler M, Knobler H, Minc-Golomb D, Bar-Peled O, Yarom R, Rotshenker S. Cell damage by excess CuZnSOD and Down's syndrome. Biomed Pharmacother. 1994; 48: 231-240.

[10] Halliwell B. Reactive oxygen species in living system: source, biochemistry and role in human disease. Am J Med. 1991; 91(3C): 14S-22S.

[11] Halliwell B. Free radicals, antioxidants and human disease: curiosity, cause or consequence? Lancet. 1994; 344: 721-724.

[12] Hoffman C, Aultman D, Pipes P. A nutrition survey of and recommendations for individuals with Prader Willi syndrome who lives in group homes. J Am Diet Assoc. 1992; 92: 823833.

[13] Hu ML, Dillard CJ, Tappel AL. In vivo effects of aurothioglucose and sodium thioglucose on rat tissue sulfhydryl level and plasma sulfhydryl reactivity. Agents Action. 1988; 25: 132138.

[14] Kaneto H, Kawamori D, Matsuoka T. Oxidative stress and pancreatic beta-cell dysfunction. Am J Ther. 2005; 12: 529533.

[15] Kaplan J, Fredrickson PA, Richardson JW. Sleep and breathing in patients with the Prader Willi syndrome. Mayo Clin Proc. Nov 1991; 66(11): 1124-1126.

[16] Knoll JH, Nicholls RD, Magenis RE, Graham JM, Lalande M, Latt SA. Angelman and Prader Willi syndromes share a common chromosome 15 deletion but differ in parental origin of the deletion. Am J Med Genet. 1989; 32: 285-290.

[17] Laurence B, Brito A, Wilkinson J. Prader Willi syndrome after 15 years. Arch Dis Child. 1981; 56: 181-186.

[18] Ledbetter DH, Riccardi VM, Youngbloom SA, Strobel RJ, Keenan BS, Crawford JD. Deletions of chromosome 15 as a cause of the Prader Willi syndrome. N Engl J Med. 1981; 304: 325-329.

[19] Lowell BB, Shulman GI. Mitochondrial dysfunction and type 2 diabetes. Science. 2005; 307: 384-387.

[20] Mark JL. Oxygen free radicals linked to many diseases. Science. 1987; 235: 529-531.

[21] Milne GL, Sanchez SC, Musiek ES, Morrow JD. Quantifi- cation of F2-Isoprostanes as a biomarker of oxidative stress. Nature Protocol. 2007; 2(1): 221-226.

[22] Nicholls RD, Knoll JH, Butler MG, Karam S, Lalande M. Genetic imprinting suggested by maternal heterodisomy in nondeletion Prader Willi syndrome. Nature. Nov 16 1989; 342(6247): 281-285.

[23] Ozcelik T, Leff S, Robinson W. Small nuclear ribonucleoprotein polypeptide N (SNRPN), an expressed gene in the Prader Willi syndrome critical region. Nature Genetics. 1992; 2: 265-269.

[24] Paffetti P, Perrone S, Longini M, Ferrari A, Tanganelli D, Marzocchi B, Buonocore G. Non protein-bound iron detection in small samples of biological fluids and tissues. Biol Trace Elem Res. Sep 2006; 112(3): 221-232.

[25] Perluigi M, Di Domenico F, Fiorini A, Cocciolo A, Giorgi A, Foppoli C, Butterfield DA, Giorlandino M, Giorlandino C, Schininà ME, Coccia R. OS occurs early in Down syndrome pregnancy: a redox proteomics analysis of amniotic fluid. Proteomics Clin Appl. Apr 2011; 5(3-4): 167-178.

[26] Perrone S, Longini M, Bellieni CV, Centini G, Kenanidis A, De Marco L, Petraglia F, Buonocore G. Early oxidative stress in amniotic fluid of pregnancies with Down syndrome. Clin Biochem. Feb 2007; 40(3-4): 177-180.

[27] Ragosta M. Rust in the pipes: the importance of oxidative stress in the pathophysiology of coronary artery disease and the valuable contribution of translational research. Atherosclerosis. 2011 Nov; 219(1): 26-7.

[28] Saugstad OD. Oxygen toxicity in the neonatal period. Acta Paediatr Scand. 1990; 79: 881-892.

[29] Saugstad OD. Mechanisms of tissue injury by oxygen radicals: implications for neonatal disease. Acta Paediatr. 1996; 85: $1-4$.

[30] Schoeller D, Levitsky L, Bandini L, Dietz W, Walczak A. Energy expenditure and body composition in Prader Willi syndrome. Metabolism. 1988; 37: 115-120.

[31] Stephenson JPB. Neonatal presentation of Prader Willi syndrome. Am J Dis Child. 1992; 146: 151-152.

[32] Valsè Pantellini G. Breve cenno sulla genesi dei tumori e sopra una eventuale terapia dei medesimi con Sali di potassio e in particolare con ascorbato di potassio. Rivista di patologia clinica. 1970; XXV(5): 219-225.

[33] Warner BB, Wispe JR. Free radical-mediated diseases in pediatrics. Semin Perinatol. 1992; 16(1): 47-57.

[34] Witko-Sarsat V, Friedlander M, Capeillere-Blandin C, Nguyen-Khoa $T$, Thu Nguyen A, Zingraff J, Jungers $P$, Descamps- Latsca B. Advanced oxidation protein products as a novel marker of oxidative stress in uremia. Kidney Int. 1996; 49: 1304-1313. 


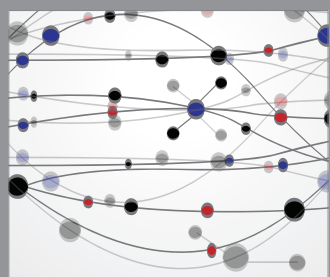

The Scientific World Journal
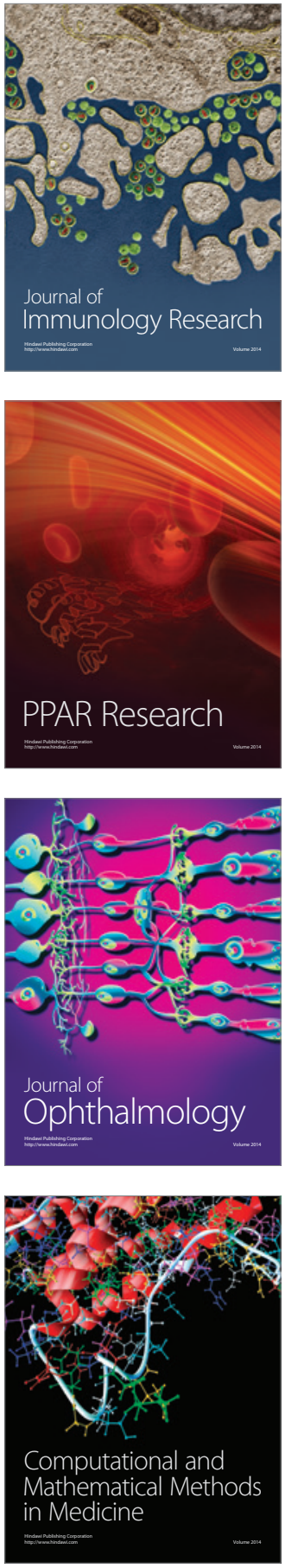

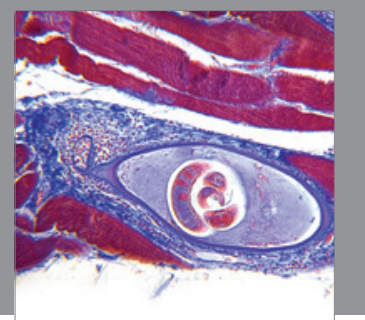

Gastroenterology

Research and Practice
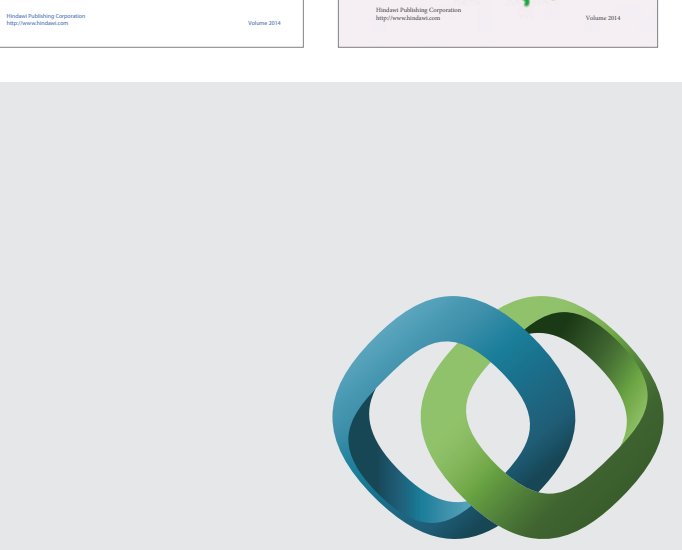

\section{Hindawi}

Submit your manuscripts at

http://www.hindawi.com
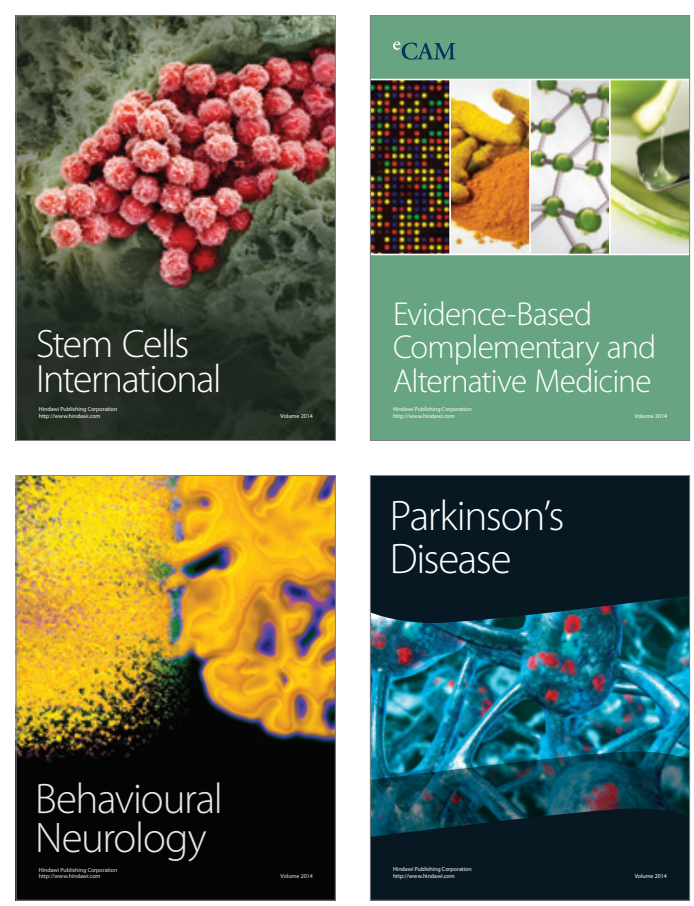

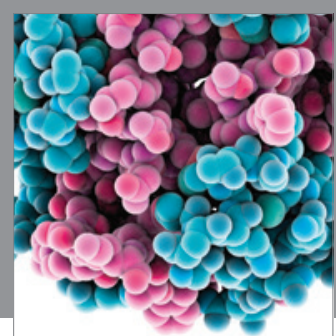

Journal of
Diabetes Research

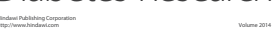

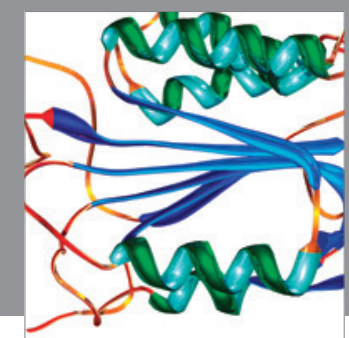

Disease Markers
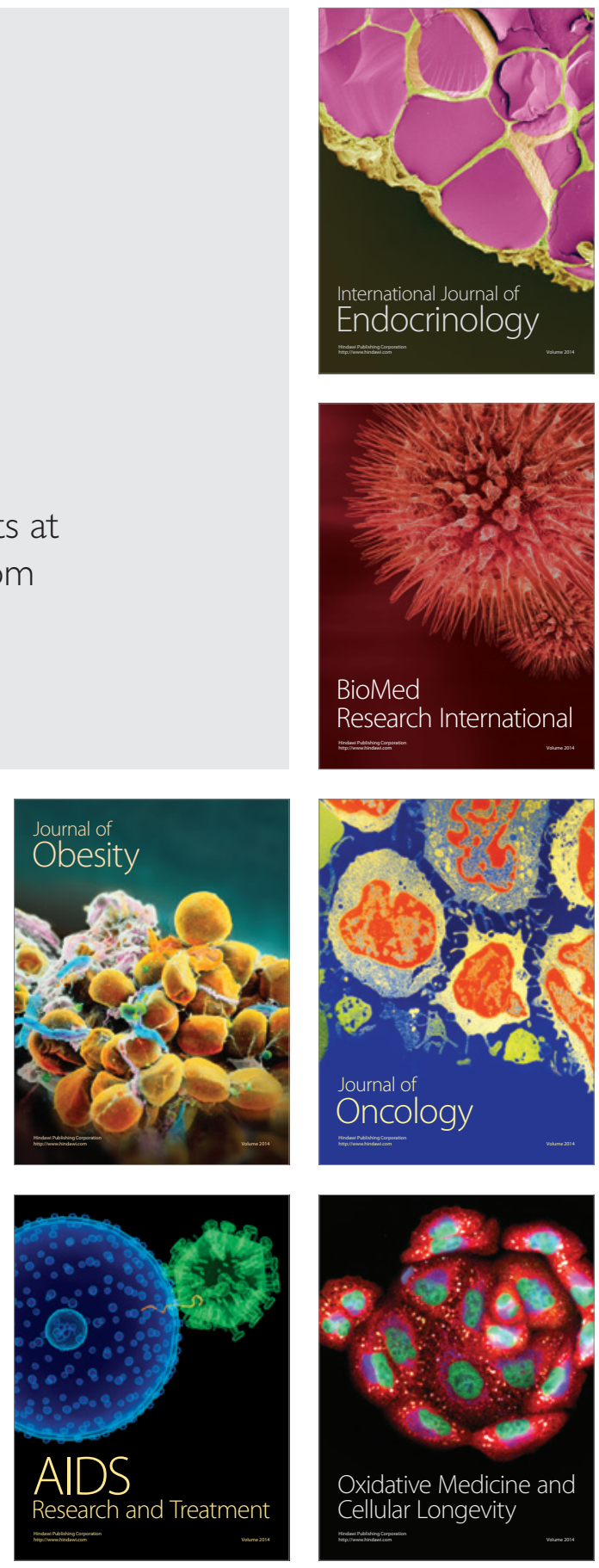